\title{
Dressing the Weightless Body: Subjective verticality and the disoriented experience of dress in microgravity
}

\section{Introduction}

As space travel increases our interactions with varying gravitational conditions, we become increasingly aware that Earth gravity (normogravity) is not the norm, but rather, the exception. A variety of other gravitational conditions will come to characterise future human experiences, including the microgravity conditions that are experienced in reduced-gravity aircraft, and in spaceflight (Beysens and van Loon 2015). In order to prepare for this postgravity age, we must identify and reconsider the ways in which gravity has dictated and constrained our approach to design (Kac 2005: 18). This includes the extent to which we, and our creative practice, are tied to the notion of the upright (Arns 2019: 19). Fashion has privileged the silhouette, at the expense of considering the other views that are presented as a consequence of weightlessness. The next 'new silhouette' will not describe a ratio of bust-towaist-to-hip as depicted in elevation view, but rather, will consider the contours of the dressed body at alternative orientations.

The author's previous contribution to his field, Spacewear: Weightlessness and the Final Frontier of Fashion (2019), introduces a range of considerations for future spacewear designers, and argues that designers must move away from the Space Age aesthetic towards an approach that engages directly with the realities of space travel, most significantly the effects of weightlessness. Since the publication of this book, spaceflight operators have taken further steps in launching commercial passengers on short-haul and long-haul spaceflights, and in employing designers to develop flight suits for their future passengers and crew. One noteworthy shift in the presentation of these new flight suit designs has been an implicit acknowledgement that the dressed, weightless bodies be oriented at a range of angles relative to each other, and to their environment. Anticipating the launch of their first commercial suborbital flights in 2020, Virgin Galactic and Under Armour presented their blue flight suits on the bodies of dancers, performing on solid ground and inside a vertical wind tunnel, so that their bodies could twist and turn in all directions (Malik 2019). Showcasing the suits in this way - not in a stable, upright position, but on summersaulting, leaping, and floating bodies recognizes one important effect of weightlessness on the clothed body of the space traveler: that it will be encountered at a range of angles and orientations. Similarly, the Design 
Museum's exhibition of Anna Talvi's Antagonist Exo. Muscle Bodysuit (2019), suspended from the ceiling, allows visitors to the Living on Mars exhibition to encounter it from below, as they might if viewing a clothed body in microgravity (see fig. 1). It is this disoriented view of the garment and the dressed body, as we might encounter in microgravity, that is the subject of this article.

Figure 1: One of the most visibly evident effects of weightlessness is the absence of a common or agreed upright, which in turn provides opportunities to showcase a designed object, or a dressed body, from all, or alternative, angles. Anna Talvi's Antagonist Exo. Muscle Bodysuit (2019) is exhibited as part of the Design Museum's Moving to Mars (18 October 2019 - 23 February 2020) in a rigged display, featuring mannequins suspended from the ceiling at a variety of angles, so that visitors to the exhibition encounter the garments from below. The display mirrors the angles at which one might encounter a weightless body during space travel. Image (C) Barbara Brownie

For future commercial spaceflight customers and spacewear designers, the presentation of the clothed body at a range of orientations offers the opportunity to showcase garments more completely, by revealing views that are not normally visible in normogravity conditions or elevation view. Further, alternative orientations enhance the wearer's space travel experience by providing visible evidence of the body's weightlessness. The appeal of space travel lies partly in the experience of weightlessness and the observation of its effects (Strickland 2012: 293), and as Susan Buckle (2017) of the UK Space Agency has observed during her own experiences of reduced gravity flight, passengers seek sensory evidence of weightlessness. The showcasing of alternative views of the clothed body, and the wearing of clothes designed with disorientation in mind, therefore has the potential to enhance experiences of weightlessness. In this social media age when the dressed body is exposed to a range of audiences, both present and remote, opportunities for expression, and the audiences for the dressed body, are multiplied and extended to include expression of the conditions that are being experienced by the wearer at the time of wearing. Dress has the potential to play a significant role in this experience, contributing to both the visual and physical sensations of weightlessness, by locating the dressed body phenomenologically and aesthetically within microgravity conditions. 


\section{Clothing and the post-gravity experience}

Kodwo Eshun (2005: 28-29) observes that gravity has 'located the human species in a condition of subjective verticality.' Taken for granted, groundedness has been an integral part of the human condition. Verticality has historically dominated human understanding of the world around us, dictating how we order physical spaces, objects, and ideas (Steyerl 2011). The Jet Age, and later the Space Age, saw the advent of aerial photography and, later, satellite imagery, which introduced new ways of looking at the Earth. Writing on the increasing ubiquity of aerial views in satellite images, Hito Steyerl (2011) concludes that 'we are growing increasingly accustomed to what used to be called a God's-eye view.' The 'stable and single point of view' that is depicted in linear perspective or elevation drawings 'is being supplemented (and often replaced) by multiple perspectives.' Steyerl examines the importance of the horizon as an orienting cue, essential in navigation, but absent when we journey underwater or into space. Aerial and satellite images introduced new views of our surroundings, and in doing so have prompted the 'departure of a stable paradigm of orientation'. This is not merely a visual phenomenon. Astronauts describe a physical 'sensation of tumbling upside down' in microgravity (Clark et al. 2015), so that the sense of one's orientation is disrupted even when artificial orientation cues are introduced. The experience of space travel prompts reflection on the extent to which the stable, elevation view is just one of many possible alternatives, that exist in different gravitational conditions than that which is common only in Earth-bound human experience.

Those who have experienced microgravity describe its physical sensations as so different from those of Earth gravity that they prompt critical reflection on the extent to which human experience has been defined in the context of gravity, and is modelled around verticality (Arns, 2019). Choreographer Kitsou Dubois (2001), who has performed in weightlessness on board reduced-gravity aircraft, describes how the weightless environment allows in her performance an 'extraordinary fluidity of movement,' in which a performer, without a center of gravity, must 'create subjective egocentric references.' Without a common understanding of up or down, weightless bodies 'build their own structures on [a] subjective axis'. On Earth, a significant part of the sensation of groundedness is the connection of ground to body via the soles of the feet. There is, notes Tim Ingold (2004: 315), a 'bias of head over heels' and 'valorization of upright posture' that arises from the regression of the status of the foot to 'a merely mechanical apparatus', while the hands, free for 'holding, feeling, and gesturing', 
maintain a connection to the intellectual functions of the human body. These ideas are challenged by space travel, where the body is no longer grounded through the feet. NASA astronaut Jerry Ross observes that, on board the International Space Station, it is frequently the hands, rather than the feet, that make contact, thus relocating the ground to beside, not below, the astronaut (Herbert 1999). Astronauts can be seen propelling themselves through the cabin with their hands, grasping and pushing off from handrails, much like a child swinging along monkey bars (NASA 2015). This is just one of several ways in which space travelers adapt by developing 'new relationships between the body references and those of the surroundings', leading to 'a new mental representation of space' (Tafforin 1996: 963).

Figure 2: Cosmonaut Yuri I. Onufrienko, Expedition Four mission commander, flanked by astronauts Daniel W. Bursch (left) and Carl E. Walz, both flight engineers, pose for an informal crew photo in the Zvezda Service Module on the International Space Station (ISS), 2001. Here, ISS crew have oriented themselves so that they are facing the camera for a group portrait, but despite having agreed a shared frontal axis (that is, facing the direction of the camera), they do not share orientation on other axes, and are therefore located at a range of orientations relative to their fellow crewmembers. Image (C) NASA.

A significant but often overlooked factor in the everyday sensation of groundedness and verticality is the way in which clothes are grounded on the body. In normogravity conditions clothing is anchored to the body wherever its contours approach horizontality, as in, for example, the shoulders or hips. From those points of contact, fabric, to varying degrees depending on its cut, weight and the structure of the garment, will drape downwards (Mei et al. 2015: 1; Hunter and Fan 2008: 7). The sensation of uprightness is therefore experienced in part through the sensation of the contact between fabric and skin at the body's horizontal surfaces, and the weight of any loose fabric that drapes down from those points of contact. When the body is positioned at alternative orientations, this too can be seen and experienced through the drape of worn fabric. When in a seated position, for example, fabric will be anchored to the horizontally oriented thighs. For aerial performers - who may be engaged in vertical dance of a vertical fashion show - the weight of a costume is distributed differently across the surface of the body when suspended at different angles. When the long body axis is oriented along the gravitational vector (in layperson's terms, upright), the weight of clothes will be felt on the body's vertical planes, including shoulders and hips, but when oriented 
with the long body axis parallel to the ground, facing downwards, the weight of clothes will be felt on the back. Clothing, or some other object or surface, is almost always in contact with the body, and the bodily location of that contact provides sensory clues as to the orientation of the body. Hence, clothing plays a part in the everyday human experience of orientation, and, on Earth's surface, clothes provide a constant reminder of gravity.

In weightlessness, clothing does not rest upon the skin, rather, it billows outwards wherever it is not constrained by cut or elasticity. Astronauts returning to Earth after long-duration visits to the International Space Station report physical discomfort from contact with everyday clothing, having become so accustomed to living without the weight of fabric against their skin (Dunn 2016; Harrington 2016). The experience of wearing clothes is defamiliarized by weightlessness to such an extent that the body must relearn how to cope with the weight of clothes on the body when returning to Earth after long-duration space travel. Without contact with the skin, clothes no longer provide reassurance of orientation, or of the presence of gravity. Annick Bureaud (2009: 4) suggests that 'weightlessness is inscribed in the limits of the self and the human body'. Without the sensation of clothes on the epidermis, skin 'stop[s] playing the role of sensor between the "interior" and the "exterior," between "me" and "what is not me"" (Bureaud 2003). The separation of body and clothes makes the external limits of the body unclear. In this way, in the weightless environment, the act of wearing is not a matter of swathing the body in cloth but, rather, blurring the divide between body and space.

\section{Dis-orientation and the fashion silhouette}

On Earth, 'the gravitational vector used as a primary spatial orientation' (Ozdemir et al. 2018). We sense that we are upright as a result of the effects of gravity on the human vestibular system, the sensory system which has evolved in Earth gravity to control balance and provide the body with a sense of its physical orientation. Weightlessness disrupts the body's ability to spatially orient itself and its parts (Bock 1998: 156). Without reliable signals from the vestibuar system, weightless bodies rely on 'the visual reference frame to control their vertical postural orientation' relative to their surroundings (Chabeauti et al. 2010: 586). Absent too is the sensation of clothes anchored on the skin, as described in the previous section. Even when there is 'haptic information' that suggests an alternative orientation, such as contact with a platform beneath the subject's feet, the weightless body will primarily rely on visual cues to determine its sense of upright orientation (Chabeauti et al. 2010: 590). In 
these microgravity conditions, a body's sense of verticality is primarily determined by visual cues such as the apparent orientation of its surroundings (surfaces that are designed to look like floors, walls and ceilings, and the orientation of objects within that environment). Visual and other cues must compensate for the sensation of being perpetually upside-down, regardless of one's actual orientation in relation to surrounding objects and surfaces. Recounting her own experience of performance in microgravity, Dubois (1994: 60) describes the body's need to find 'a new system of references' with which to orient itself. She notes that 'an upward or downward movement of the eyes' locates objects and surfaces in or out of alignment with 'body's longitudinal axis, rather than the gravitational vertical'. In microgravity, therefore, verticality is subjective: 'orientation of an object is a relative concept, and changes with regard to the viewer' (Cornelis et al. 2016: 2).

In fashion, the term silhouette is used to describe the shape of the body or garment as if reduced to a flat representation of the front, back, or side views. Clothes are often defined by their silhouette - the vertical contours of the garment that give it a distinct ratio of bust to waist to hip to hem. Moreover, the notion of the silhouette has been particularly important in defining historical developments in fashion. Silhouette, more so than other features of womenswear, has been used to represent the evolution of fashion, with developments defined in terms of silhouette. Early fashion illustrations frequently depict front and back views, flattened onto the page. The term 'the new silhouette' has been used to introduce developments in fashion for more than a century. Vogue used the phrase in 1905 (April: vi) to describe the clearly defined waist of the latest Parisian gowns, and then again in 1913 (April: 44) to herald the emergence of the modernist, tubular silhouette. As the shape of womenswear continued to evolve throughout the twentieth century, each new era was welcomed with a celebration of another 'new silhouette,' from the New Look (September 1947: 141) to the 'slim skirts, narrow pants, and asymmetric gowns' of Fall 1996 (September: 578). Prioritization of the front, back, and side views has led to a history of enhancements that distort the silhouette as seen from these views, including enhancement of the bust or hips in contrast to the waist in side or front view, or the rear in contrast to the waist in side view (as in the bustle). Koda (2002: 11) describes clothes as a tool for 'the reformation of the body', but his selection of examples more accurately describes reformation of the silhouette, as the body parts that he singles out for study (neck, chest, waist, hips) are enhanced in ways that contrast these with other dimensions when worn by the body in anatomical position and viewed in elevation. 
The silhouette, as the term is used in fashion, is drawn from the frontal plane of a body in anatomical position, though fashion also concerns itself with the sagittal plane - the side view. Both of these views are akin to orthographic projections of the front and side of an object (referred to here as the front and side silhouette). These views are the focus of both verbal and visual description of garments, with fashion illustrations commonly presenting these same front, rear, or side views. It is reasonable to prioritize these elevation views, and hence the silhouette, of a garment when clothes are most commonly encountered in an upright position, relative to their surroundings, as, 'in most everyday conditions [on Earth's surface], the viewer-centered reference frame coincides with the environmental reference frame' (Cornelis et al. 2016: 2) and the viewer's orientation is likely to be in alignment with that of any dressed bodies that she encounters. However, footage from microgravity environments reveals that elevation views of the human body are rarely, if ever, encountered in space. Bodies are more likely to be encountered in sections and at angles that reveal shoulders or legs before the torso. Footage of the ISS depict astronauts and cosmonauts propelling themselves through the cabin with their hands. Rather than parading past an audience as would a model on a catwalk, revealing the garment from front to back at the model parades and then turns, bodies glide past so that garments are revealed gradually from shoulder to toe, and in distant views, either the soles of the feet or the overhead view of the shoulders are often seen. In fig. 3, astronaut Ellen Ochoa is encountered shoulder-first, as she propels herself head-first toward the camera. Such views are typical of those photographed or video recorded when astronauts and cosmonauts are travelling through the ISS, and highlight the extent to which the frontal view of a garment is unavailable to the viewer.

Figure 3: Astronaut Ellen Ochoa floats through the tunnel that connected the STS-96 crew to the International Space Station (ISS) for several days in late May and early June 1999. Ochoa's angle of approach leads to an overhead view of her shoulders, in which shoulder seams, collar, and sleeves, are the most visible parts of her garment. Image (C) NASA.

Footage of dressed bodies on parabolic flights also provide some indication of the extent to which the clothed, weightless body is encountered at uncommon angles. Promotional video footage produced by reduced gravity flight operator Zero G shows passengers twisting laterally, summersaulting, or drifting at a range of orientations, and, frequently, needing to be 
manually reoriented and stabilized by cabin crew before the return to normogravity. Moreover, the orientation of one passenger is never aligned with that of another, meaning that each passenger encounters another from a different viewpoint. Each passenger will present a different set of bodily contours, and a different section or view of the Zero G branded flight suit. Weightless bodies can be seen in everyday clothing in the music video for OK Go's Upside Down \& Inside Out, which was filmed over the course of twenty-one flights on an S7 reduced gravity aircraft (Howarth 2016). Throughout the video, the performers attempt to remain oriented vertically, relative to the camera, by grasping onto the handrails, seats, or other fixed parts of the aircraft's interior. Whenever they are not stabilized in this way, the performers' bodies revolve at various directions away from the camera. The bodies rarely share the same orientation, so that we may see the profile view of one performer while we see an aerial view of another (see fig. 4). As they move from the front to the back of the cabin, or vice versa, they do so not by walking, but rather, by diving or floating with their bodies parallel to the floor. The overall affect is disorienting for both the performers and the viewers. The 360-degree view of each body that is revealed as they tumble through the interior space has the consequence of revealing every angle and surface of the performers' clothing, including the soles of their shoes. ${ }^{i}$ Fashion's preoccupation with the silhouette, as it is used to represent the side or front elevation view, has led designers to neglect the features of a garment that are visible from these other angles. Little or no consideration is given to the alternative views that could be encountered when a garment is encountered from above or below, as is likely in weightlessness.

Figure 4: Members of the band OK Go float around the cabin of a reduced gravity aircraft. Here they are seen at a variety of different orientations relative to the cabin and to each other. As a result, we encounter their clothes from a variety of different angles, including the top of their shoulders, as if viewed from above, and soles of their feet, as if viewed from below.

The likelihood of encountering the dressed body at alternative orientations offers both challenges and opportunities to spacewear designers. First and foremost, it requires designers to revisit one of the most fundamental principles of design, and abandon their tendency to imagine and depict garments in elevation view. Instead, they must consider the appearance of garments from all orientations, including above, below, and all angles in between. The shapes presented by the weightless body can therefore not easily be mapped onto the silhouette, as it 
is currently understood. The author's survey of the views of bodies in NASA footage of the International Space Station reveals that, in microgravity, the silhouette is replaced by less symmetrical, sometimes more angular shapes. The difference in girth of the waist and hips, or bust and hips - the ratio that we use to determine silhouette - is not often visible. The knees and elbows become more prominent, and, due to the parts body being arranged so that they are not in vertical alignment with the head, the body is not presented as if aligned to a plane (see fig. 5). These newly realized shapes, which can be exploited by fashion designers as alternative silhouettes, create new possibilities for fashion and the presentation of the clothed body.

Figure 5: 3D scans depicting an artist's model in neutral body posture, at a range of orientations presented by the weightless body in microgravity, based on a survey of publicly available NASA video footage of the International Space Station. In these positions, the ratio of bust-to-waist-to-hip is rarely visible, due to the angle and posture of the body, that does not allow the presentation all three of these measurements simultaneously. A three-dimensional CAD model - in this case, a 3D scan of a posed mannequin - is a useful design tool when attempting to visualize the appearance of a clothed body in microgravity, allowing the designer to rotate the model to any orientation. Image (C) Barbara Brownie

These alternative presentations of the body are not solely the result of the weightless body's orientation, but also its neutral posture. The weightless body is not encountered outstretched, as in the atomically posture of a standing mannequin or illustrations of a fashion silhouette. Instead, the spine and joints bend forwards, as if in a slouched, semi-seated position (Dominoni 2005: 6). At alternative orientations, and in neutral posture, the space traveler's garment is rarely seen as one piece, but rather, is segmented, as the body, folded into neutral posture, locates its limbs out of alignment with the body. As footage from microgravity environments suggests, parts of the garment that are most likely to be encountered are the shoulders (as if viewed from above), the rear (seat), the shins, and the soles of the shoes. These alternative views expose parts of the garment that are not commonly prominent when viewing a garment from the front, side, or rear view, such as the cuff of the sleeves, or the hem of a skirt. When viewed from the front or side, these hems appear horizontal, but when viewed as if from below, they can be revealed as circles. Thus, the designer can exploit potential for a range of new shapes that are not part of the silhouette, but that that have the 
same potential to be distorted or accentuated, and to become some of the defining shapes of a garment.

These alternative views expose internal parts of the garment that are, in Earth gravity, designed to be unseen, such as the lining or other reverse side of cloth. NASA footage of astronauts reveals views that include the internal lining of shorts and trousers. Footage of passengers on board an Astrax reduced gravity flight show the underside of a skirt as its wearer tumbles through the cabin and her skirt billows outwards, potentially compromising the wearer's modesty. Here too, there are challenges and opportunities. By exposing normally overlooked parts of the garment, these alternative views of the clothed body highlight gaps in fashion design practice. Where designers have focused their attention on the orthographic view, and the areas of the garment that are prominent in that view, they have neglected to explore other potential sites for creative expression. Design that enhances these views of the garment has the potential to contribute to the aesthetic pleasures of weightlessness, by emphasizing the difference in the experience of viewing a garment in different gravitational conditions.

\section{Conclusion: The post-gravity body}

As we enter the new commercial space age, and begin our journey towards becoming an interplanetary species, we will more commonly experience a variety of different gravitational conditions. Those who have experienced weightlessness describe the realisation that, although it has been taken for granted throughout human history, Earth gravity, or normogravity, is not normal in the wider universe. Gravity has significantly impacted our approach to design, our interactions with designed object, and indeed, more broadly, has contributed to the experience of being human. For Kodwo Eshun (2005: 29), post-humanism can be achieved through the experience of weightlessness, that enables one to re-experience gravity as a 'peculiarity'. 'Even a temporary stay in states of weightlessness... changes one's relation to the body and its terrestrial possibilities' (Ryklin 2005: 15), and so for designs who work as closely with the body as fashion designers and dressmakers, it will be vital to take a critical view of design practices that have been developed within Earth gravity. Although we cannot predict what future space travellers will choose to wear, we can challenge some of the assumptions made by Earthwear designers, and identify ways in which clothing will be encountered differently in microgravity. 
Observations by Annalisa Dominoni (2015) and Mark Timmins (2011) illustrate the need for Earthwear to be adapted or redesigned for the weightless environment, and for the introduction of a dedicated field of Spacewear design. Approaches to design that are taken for granted on Earth will need to be reconceived for the weightless environment. Designers will be required to rethink many of the assumptions about weight and balance upon which gravity-bound design is founded, leading to new approaches to product and fashion design. Spacewear design will need to develop well beyond the space-themed fashion of the Space Age, which signified space travel through the use of modern textiles and clean, streamlined silhouettes (Baldaia 2015). In order to prepare for the real possibility of commercial space travel, the fashion industry must acknowledge that it is not sufficient to simply signify spaceflight, and must begin to produce clothing that is more appropriate for the environments encountered on board spaceflights and reduced gravity aircraft. Challenges and considerations include the absence of upright, and the neutral posture of the weightless body. The disoriented body challenges designers to abandon their preoccupation with silhouette, and to revisit some of their most fundamental assumptions about clothes - that clothes and the body have up an upright orientation, and that clothes should be designed, depicted, and presented primarily in orthographic view. For the designer or the wearer, microgravity provides an opportunity to showcase the design of a garment from all possible angles, and to explore the alternative shapes presented by the body when viewed from these angles. The potential for a garment to be viewed at any orientation introduces the need to consider the design of otherwise unseen or overlooked views, such as the view from above or below, including not only the parts of the garment that are exposed from these new views, but also the overall shape that replaces the orthographic silhouette at other viewing angles.

\section{References}

Arns, Inke (2019), 'Zero Gravity, Anti-Mimesis and the Abolition of the Horizon: On Cosmokinetic Cabinet Noordung's “Postgravity Art”,' Leonardo, 52: 1, pp. 17-22. Baldaia, Suzanne (2015), 'Space Age Fashion', Berg Fashion Library, London: Berg. Basalla, George (1982), 'Transformed Utilitarian Objects', Winterthur Portfolio, 17: 4, pp. 183-201. 
Battaglia, Debbora (2012), 'Coming in at an Unusual Angle: Exo-Surprise and the Fieldworking Cosmonaut', Anthropological Quarterly, 85: 4, pp. 1089-1106.

Beyens, Daniel A. and van Loon, Jack J. W. A. (2015), 'The Space Gravity Environment', in D. A. Beysens and J.J.W.A. van Loon (eds), Generation and Applications of ExtraTerrestrial Environments on Earth, Delft, The Netherlands: River, pp. 5-10.

Bock, Otmar (1998), 'Problems of sensorimotor coordination in weightlessness', Brain Research Reviews, 28: 1-2, pp. 155-160.

Buckle, Susan (2017), telephone interview, 5 May.

Bureaud, Annick (2003), 'Reasons for a Symposium', paper presented at Visibility Legibility of Space Art. Art and Zero G. : the experience of parabolic flights, Maison Européenne de la Photographie, Paris, 4-5 October, https://www.olats.org/space/colloques/artgravitezero/t_Bureaud_en.html. Accessed 28 October 2019.

Bureaud, Annick (2009), 'Kitsou Dubois and the Weightless Body', Journal of Multimedia, 16: 1, pp. 138-147.

De Caro, Stefano, and Reeves, Adam (2000), 'Rotating objects to determine orientation, not identity: Evidence from a backward-masking/dual-task procedure', Perception \& Psychophysics, 62: 7, pp. 356-1366.

Chabeauti, P., Vaugoyeau, M. \& Assaiante, C. (2010), 'Is vertical postural orientation in weightlessness correlated with the subjects' perceptual typology?', Gait \& Posture, 32: 4, pp. 586-591.

Chowdhury, A. A. (2016), 'Foot Restraint Version Used during the Skylab Missions', NASA Hardware archives. https://lsda.jsc.nasa.gov/scripts/hardware/hardconfi g.aspx?hardware_index=2057\&hardware_id=1044. Accessed 1 February 2017.

Cornelis, E.V.K., Van Doorn, A.J. \& Wagemans, J. (2016), 'Reference Frames and 3-D Shape Perception of Pictured Objects: On Verticality and Viewpoint-From-Above', $i$ Perception, 7: 3, pp. 1-42.

D’Aloia, Adriano (2012), 'The Intangible Ground: A Neurophenomenology of the Film Experience', European Journal of Media Studies, 1: 2, pp. 219-239 . 
de Winkel, K.N., Clément, G., Groen, E.L. \& Werkhoven, P.J. (2012), 'The perception of verticality in lunar and Martian gravity conditions', Neuroscience Letters, 529: 1, pp. 7-11. Dominoni, Annalisa (2005), 'VEST. Clothing Support System On-Orbit Validation', paper presented at the 25th International Conference on Environmental Systems (ICES), Rome, Italy, 11-14 July, http://hdl.handle.net/11311/255047. Accessed 5 July 2015.

Dominoni, Annalisa (2015), For Designers with their Heads beyond the Clouds, Santarcangelo di Romagna: Maggioli Editore.

Doyle, Denise (2015), 'Out of this world: exploring embodiment and space through artistic processes and practice', International Journal of Performance Arts and Digital Media, 11: 1, pp. 1-17.

Dubois, Kitsou (1994), 'Dance and Weightlessness: Dancers' Training and Adaptation Problems in Microgravity', Leonardo, 27: 1, pp. 57-64 .

Dubois, Kitsou (2001), 'Dance and Weightlessness' ('Danse et apesanteur'), in E. Chardronnet (ed.), Exit Gravity ('Quitter la gravit'), Association des Astronautes Autonomes. http://www.lyber-eclat.net/lyber/aaa/danse.html. Accessed 4 August 2016. Dunn, Marcia (2016), 'A Year in Space: Aching Joints and the Weird Feeling of Clothing Touching Your Skin', Global News, 4 March, https://globalnews.ca/news/2558552/ayear-inspace-aching-joints-and-the-weird-feeling-of-clothing-touching-your-skin/. Accessed 27 January 2018.

Eshun, Kodwo (2005), 'On the Use and Abuse of Microgravity for Life', in R. La Frenaisand N. Triscott (eds), Zero Gravity: A Cultural Users Guide, London: The Arts Catalyst, pp. 28-31.

Harrington , R. (2016), ‘A Famous Astronaut Says His Body Still Hurts after Spending a Year in Space', Tech News, 8 June, http://uk.businessinsider.com/scott-kelly-spacetraveleffects-human-body-2016-6?r=US\&IR=T. Accessed 15 December 2017.

Herbert, D. (dir.) (2001), 'Space Wear', That NASA Show, Episode 2 Show B, Version D, https://archive.org/details/space_wear. Accessed 9 August 2015.

Howarth, Dan (2016), 'OK Go performs in zero-gravity for Upside Down \& Inside Out music video', Dezeen, 20 February, https://www.dezeen.com/2016/02/20/ok-go-performs-inzero-gravity-for-upside-down-inside-out-music-video-interview-trish-sie/. Accessed 24 October 2019. 
Hunter , L., and J. Fan (2008), 'Measuring and Predicting Fabric and Garment Drape', in Catherine Fairhurst (ed.), Advances in Apparel Production, Cambridge, UK: Woodhead, pp. $7-25$.

Ingold, Tim (2004), 'Culture on the ground', Journal of Material Culture, 9: 3, pp. 315-340. Jones, Sue Jenkyn (2011), Fashion Design, London: Laurence King.

Jolicouer, P. and McMullen, P.A. (1992), 'Reference frame and the effects of orientation on finding the tops of rotated objects', Journal of Experimental Psychology: Human Perception and Performance, 18: 3, pp. 807.

Kac, Eduardo (2005), 'Against Gravitropism: Art and the Joys of Levitation,' in R. La Frenaisand N. Triscott (eds), Zero Gravity: A Cultural Users Guide, London: The Arts Catalyst, pp. 18-25.

Koda,Harold (2002), Extreme Beauty: The Body Transformed, New York: The Metropolitan Museum of Art.

La Frenais, Rob (2005), 'An Introduction to Vertigo', in R. La Frenaisand N. Triscott (eds), Zero Gravity: A Cultural Users Guide, London: The Arts Catalyst, pp. 8-10.

Malik, Tariq (2019), 'Virgin Galactic Unveils Under Armour Spacesuits and 'Spacewear' for Space Tourist Flights', Space.com, 17 October. https://www.space.com/virgin-galacticunveils-under-armour-spacesuits-spaceshiptwo.html. Accessed 18 October 2019.

Mei , Z. , W. Shen, Y. Wang, J. Yang, T. Zhou, and H. Zhou (2015), 'Unidirectional Fabric Drape Testing Method', PLoS ONE, 11: 10, pp. 1-15 .

NASA (2015), 'International Space Station Tour', http://www.nasa.gov/mission_pages/station/main/suni_iss_tour.html. Accessed 16 July 2016. Ozdemir, R.A., Goel, R., Reschke, M.F., Wood, S.J. \& Paloski, W.H. (2018), 'Critical Role of Somatosensation in Postural Control Following Spaceflight: Vestibularly Deficient Astronauts Are Not Able to Maintain Upright Stance During Compromised Somatosensation', Frontiers in Physiology, 9, pp. 1680.

Rock, Irvin (1973), Orientation and Form, New York: Academic Press. Rock, Irvin (1975), An Introduction to Perception, New York: Macmillan. Ryklin, Mikhail (2005), 'Being There', in R. La Frenaisand N. Triscott (eds), Zero Gravity: A Cultural Users Guide, London: The Arts Catalyst, pp. 14-17. 
Siede, Caroline (2015), 'On Apollo 13's 20th anniversary, a look at how they made the film so realistic', $A V$ News, 30 June, https://news.avclub.com/on-apollo-13-s-20th-anniversary-alook-at-how-they-mad-1798281369. Accessed 16 July 2019.

Strickland , P. (2012), 'Do Space Hotels Differ from Hotels on Earth?' Journal of Hospitality Marketing and Management, 21: 8, pp. 897-908 .

Tafforin, Carole (1996), 'Initial moments of adaptation to microgravity of human orientation behavior, in parabolic flight conditions', Acta Astronautica, 38: 12, pp. 963-971.

Timmins, Mark (2011), 'From Spacesuits to Space Couture: A new aesthetic,' in Ulrike Landfester, Nina-Louisa Remuss, Kai-Uwe Schrogl, Jean-Claude Worms (eds), Humans in Outer Space - Interdisciplinary Perspectives, New York: Springer, pp. 183-203.

\footnotetext{
${ }^{\mathrm{i}}$ Here, there is an opportunity for design innovation in footwear design. While footwear design on Earth is constrained by the need for the sole to provide stable contact with the Earth (and for that reason, most commonly takes the form of a platform), there is scope for space footwear to make alternative uses of the soles, either as functional surfaces or sculptural appendages. The functional potential of the sole of the shoe has been recognised by NASA, who, in 1973-74 provided astronauts with shoes bearing triangular cleats on their soles, that would lock into a grid system on the internal walls of Skylab (Chowdhury 2016), and more recently Edward Howell (2010) in his proposed design for Zero Gravity Prehensile Footwear.
} 